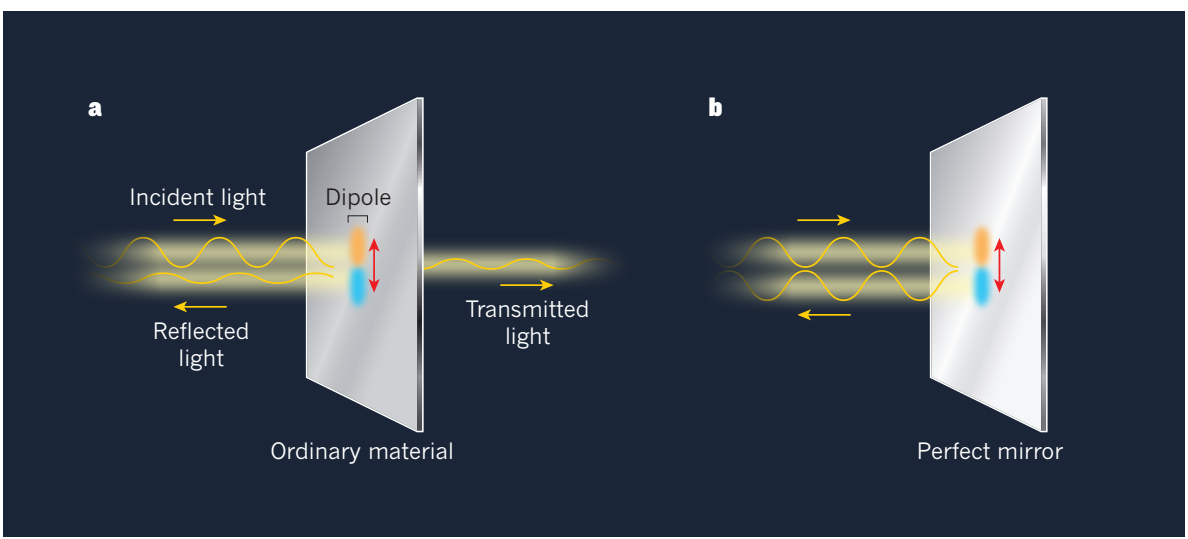

Figure 1 | A conventional material versus a perfect mirror. a, When a wave of light hits a thin film of an ordinary material, it produces electric dipoles - separations between positively charged (orange) and negatively charged (blue) particles. These dipoles oscillate (red arrow) at the same frequency as that of the incident light. They re-radiate light waves in both the forward and backward directions, which gives rise to transmission and reflection, respectively. $\mathbf{b}$, By contrast, in a hypothetical perfect mirror, there is no absorption or transmission, and the incident light is reflected entirely. Back et al. ${ }^{3}$ and Scuri et al. ${ }^{4}$ report near-perfect mirrors made of a single layer of the material molybdenum diselenide.

interference. Owing to conservation of energy, the incident light would be reflected entirely, and the material would act as a perfect mirror (Fig. 1b). This holds true even when the material comprises a single layer of atoms, provided that the oscillating dipoles are being driven at their resonance frequency.

Although theoretical studies have suggested that such conditions could be realized in a 2D array of ultracold atoms $\mathrm{s}^{5,6}$, the authors demonstrate near-perfect mirrors in a solid-state system. They use a single layer of molybdenum diselenide, which is a semiconductor and belongs to a family of materials known as the transition-metal dichalcogenides. In such materials, the oscillating dipoles generated by the incident light are excitons ${ }^{7}$ - bound pairs of an electron and a hole (the absence of an electron). The more tightly bound the excitons are, the larger the radiative damping will be, and the more perfectly the mirror will behave. Previous experimental work has shown that the exciton binding in single-layer transition-metal dichalcogenides is extremely strong $^{8-10}$, which results in a rate of radiative damping that is much greater than that of conventional semiconductors.

Back et al. and Scuri et al. fabricated highquality samples of single-layered molybdenum diselenide by encapsulating the material in atomically thin, inert films of hexagonal boron nitride, and then carried out their experiments at a low temperature (4 kelvin). Under these conditions, the authors show that radiative damping of the excitons is the dominant process. They demonstrate mirrors that can reflect a considerable proportion of the incident light - up to $85 \%$ in Scuri and colleagues' study - at the exciton resonance frequency of the material.

Although the authors' near-perfect mirrors work only in light from a narrow range of the electromagnetic spectrum (in the vicinity of the resonance frequency), the two studies open up intriguing possibilities for the fields of nanophotonics and quantum optics. For instance, quantum nonlinear optics requires strong interactions between photons at the single-photon level, which is difficult to achieve in conventional materials. The authors' work shows that quantum nonlinear optics could be realized in single-layer transition-metal dichalcogenides because of the extremely strong light-matter interactions that can be achieved $^{11}$.

The authors also demonstrate that the application of a voltage causes the mirrors to switch from being highly reflective to highly transparent. Such mirrors could therefore be used as light modulators, or as other

\section{PLANT BIOLOGY}

\section{ALEXANDER CHRISTMANN \& ERWIN GRILL}

$\mathrm{B}$ ecause plants cannot move to escape unfavourable conditions, they must continuously monitor environmental cues to survive when conditions change. Plants can sense interactions with other organisms, such as bacteria, and can monitor light conditions across the spectrum, from ultraviolet to far red. The molecular mechanisms that facilitate those capacities are well understood. But how plants sense drought, cold and salt has reconfigurable components, in optical and optoelectronic systems. Moreover, the excitons in single-layer transition-metal dichalcogenides have a feature known as the internal-valley degree of freedom ${ }^{7}$, which might enable the mirrors' reflectance to be controlled purely by varying the polarization of the incident light.

About a decade ago, during the early stages of research on 2D materials, many scientists were asking whether a single layer of atoms could be highly reflective. Thanks to Back et al. and Scuri et al., we now know that the answer is yes. $\square$

Kin Fai Mak and Jie Shan are in the Laboratory of Atomic and Solid State Physics and the School of Applied and Engineering

Physics, Cornell University, Ithaca,

New York 14853, USA.

e-mails:kinfai.mak@cornell.edu; jie.shan@cornell.edu

1. Novoselov, K. S. et al. Science 306, 666-669 (2004).

2. Casiraghi, C. et al. Nano Lett. 7, 2711-2717 (2007).

3. Back, P., Zeytinoglu, S., ljaz, A., Kroner, M. \& Imamoğlu, A. Phys. Rev. Lett. 120, 037401 (2018).

4. Scuri, G. et al. Phys. Rev. Lett. 120, 037402 (2018).

5. Bettles, R. J., Gardiner, S. A. \& Adams, C. S. Phys. Rev. Lett. 116, 103602 (2016).

6. Shahmoon, E., Wild, D. S., Lukin, M. D. \& Yelin, S. F. Phys. Rev. Lett. 118, 113601 (2017).

7. Mak, K. F. \& Shan, J. Nature Photon. 10, 216-226 (2016).

8. He, K. et al. Phys. Rev. Lett. 113, 026803 (2014).

9. Chernikov, A. et al. Phys. Rev. Lett. 113, 076802 (2014).

10. Ye, Z. et al. Nature 513, 214-218 (2014).

11.Zeytinoğlu, S., Roth, C., Huber, S. \& Imamoğlu, A. Phys. Rev. A 96, 031801 (2017).

This article was published online on 9 April 2018.

\title{
Peptide signal alerts plants to drought
}

It is thought that plants sense water availability in the soil as a way of anticipating drought. The identification of a peptide expressed when water is scarce offers a chance to unravel the underlying molecular mechanism. SEE LETTER P.235

remained an enigma ${ }^{1}$. On page 235 , Takahashi et al. ${ }^{2}$ report the identification of a peptide that is generated in response to a water deficit in plants.

Drought, cold or salty conditions can affect a plant's water status. Such conditions result in the synthesis ${ }^{3}$ of the hormone abscisic acid (ABA), which can regulate the plant's water levels. Stomatal pores in leaves enable plants to take up the carbon dioxide required for photosynthesis, but water vapour can escape through them. ABA can trigger a reduction in 
how fully stomatal pores are opened ${ }^{4}$, helping to conserve water.

The molecular basis of the link between water deficit and the induction of ABA synthesis has been a mystery. Using the plant Arabidopsis thaliana as a model system, Takahashi and colleagues investigated whether members of the CLE family of secreted peptides might have a role in this process. There are more than 30 members of this family, and they are generated by an enzyme-mediated cleavage event. These peptides are involved in diverse biological processes ${ }^{5}$. For example, CLAVATA 3 controls the fate of stem cells, and TDIF regulates the formation of the vasculature, the water-transport tissues of plants ${ }^{6}$.

Takahashi et al. tested 27 CLE peptides for their ability to stimulate ABA synthesis, which is known $^{7}$ to occur in the vasculature in response to drought. Enzymes called NCEDs cleave a carotenoid precursor molecule in the pathway that gives rise to $\mathrm{ABA}$, and the expression of the gene NCED3 is induced by drought ${ }^{3}$. The authors administered CLE peptides to the roots of plants, and monitored whether this treatment induced NCED3 in leaves. They found that, at low levels of peptide application, only CLE25 was active in regulating NCED3 expression. CLE25 treatment resulted in an increase in ABA levels and a decrease in stomatal opening. The CLE25 gene was rapidly expressed in roots in response to drought, and CLE25-deficient mutant plants failed to induce NCED 3 expression in response to dehydration. The formation of CLE25 in the root or shoot was enough to induce NCED3 in response to dehydration.

The authors tested groups of receptors known to recognize CLE peptides, and identified the receptor proteins BAM1 and BAM3 as being necessary for CLE25-induced responses. A series of grafting experiments clarified how this system works. If roots containing mutations in both the BAM1 and BAM 3 genes were grafted to wild-type shoots, the application of CLE25 to the plant's roots led to NCED3 expression in the shoot. However, if the plant was a graft between wild-type roots and shoots that had mutations in both the BAM1 and $B A M 3$ genes, $N C E D 3$ was not expressed in the shoot in response to root application of CLE25.

These results are consistent with a model in which CLE25 expressed in the roots can travel to the leaves and bind to BAM1 or BAM3 (Fig. 1). The authors confirmed this pattern of CLE25 mobility by using a mass-spectrometry technique to identify CLE25 peptides that had travelled from the root to the leaf. Little is known about how CLE peptides travel within the plant, and not all such peptides travel as far as CLE25: CLAVATA3 moves only a few layers of cells ${ }^{8}$, for example.

Takahashi and colleagues' findings open up potential avenues for determining the longsought molecular events that occur when a water deficit is initially sensed. The steps leading to CLE25 expression in response to dehydration are unknown, and their discovery
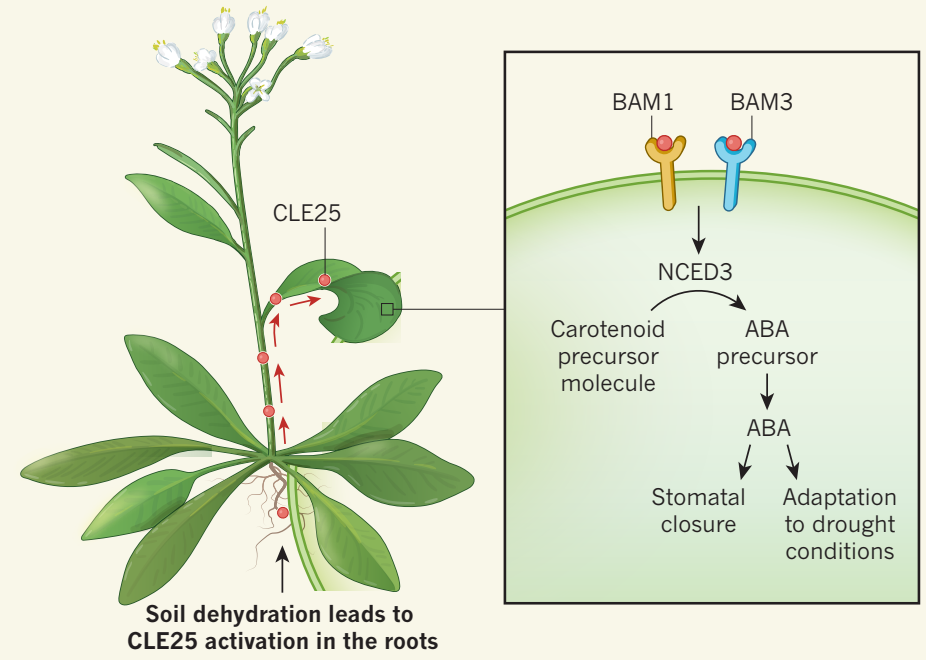

Figure 1 | A peptide aids a plant's response to drought. In drought ${ }^{3}$, plants generate the hormone $A B A$, which can help to regulate plant water levels using processes such as the closure of stomatal pores, through which water escapes from leaves. However, the steps that occur between a plant sensing drought and the production of ABA were previously unknown. Using the plant Arabidopsis thaliana, Takahashi et al. ${ }^{2}$ report that the peptide CLE25 is activated in response to drought and is a mobile signal, moving from the roots to the leaves. The authors propose BAM1 and BAM3 proteins as receptors for CLE25, and their results indicate that interactions of CLE25 with these receptors leads to expression of the carotenoidcleaving enzyme NCED3. The action of this enzyme generates an ABA precursor molecule ${ }^{3}$, which is converted to the active ABA signal, facilitating changes that help the plant to cope with a water shortage.

would shed light on this matter. And many questions remain about how CLE25 levels are regulated. How does the presumed cleavage of CLE25 occur? Chemical modifications to CLE25, including the hydroxylation of proline amino-acid residues and possibly the addition of sugar groups, might be crucial for its activity ${ }^{5}$. Whether such modifications are necessary for the function of CLE25 in the drought-sensing process should be investigated.

The molecular mechanism of CLE25 action might be evolutionarily conserved in other plants. The results of Takahashi and colleagues suggest that the CLE25 peptide is generated by the enzymatic cleavage of a precursor protein that generates a 12-amino-acid peptide. In our own analysis of gene sequences, we note that the sequence of this CLE25 peptide in A. thaliana is identical to that of many other species, including beet (Beta vulgaris), poplar (Populus trichocarpa), rice (Oryza sativa) and maize (corn; Zea mays).

Previous analysis ${ }^{9}$ revealed that water deficit can result in tension in the vasculature that can serve as a signal for ABA induction. Transport of CLE25 from the roots to the leaves is likely to be much slower than the immediate relay of this tension cue. Whether this cue and CLE25 act together or independently needs to be addressed. BAM1 and BAM3 are linked to the maintenance of meristem structures, which contain stem cells, and to vasculature development ${ }^{10}$. Whether these receptors use the same signalling pathways for those developmental processes as the ones used in this drought response also awaits further analysis.

The authors' identification of this role for CLE25 provides an intriguing insight into the regulatory interaction network that plants use to optimize their performance and viability under drought conditions. Water deficit is the major limiting factor for crop yields, and an improved understanding of the molecular strategies used by plants to cope with this environmental challenge ${ }^{11}$ might reveal ways of boosting crop resilience and ensuring stability in the future.

Alexander Christmann and Erwin Grill are in the Department of Plant Sciences, Technical University of Munich, 5354 Freising, Germany.

e-mails:christma@wzw.tum.de; erwin.grill@wzw.tum.de

1. Zhu, J.-K. Cell 167, 313-324 (2016).

2. Takahashi, F. et al. Nature 556, 235-238 (2018).

3. Nambara, E. \& Marion-Poll, A. Annu. Rev. Plant Biol. 56, 165-185 (2005).

4. Kim, T.-H., Böhmer, M., Hu, H., Nishimura, N. \& Schroeder, J. I. Annu. Rev. Plant Biol. 61, 561-591 (2010).

5. Matsubayashi, Y. Annu. Rev. Plant Biol. 65, 385-413 (2014)

6. Ito, Y. et al. Science 313, 842-845 (2006).

7. Endo, A. et al. Plant Physiol. 147, 1984-1993 (2008)

8. Lenhard, M. \& Laux, T. Development 130 , 3163-3173 (2003).

9. Christmann, A., Grill, E. \& Huang, J. Curr. Opin. Plant Biol. 16, 293-300 (2013).

10. DeYoung, B. J. et al. Plant J. 45, 1-16 (2006).

11. Yang, Z. et al. Proc. Natl Acad. Sci. USA 113, 6791-6796 (2016)

This article was published online on 4 April 2018. 